Bio - grafia. Escritos sobre la Biología y su Enseñanza. ISSN 2027

Edición Extraordinaria. p.p. 1147- 1154

Memorias del VIII Encuentro Nacional de Experiencias en Enseñanza de la Biología y la Educación Ambiental. III Congreso Nacional de Investigación en Enseñanza de la Biología.

\title{
CUANDO LOS DOCENTES SE CONVIERTEN EN AUTORES DE TEXTO
}

WHEN TEACHERS WILL BECOME AUTHORS OF TEXT

CARÍSSIMO, MARÍA SOL'; GIORDANO, MARÍA INÉS²

1Profesora de la EGB 3 y Polimodal en Biología. Licenciada en la Enseñanza de la Biología,

E-mail: msc_biologia@hotmail.com

2Profesora de Biología. Licenciada en Ciencias Biológicas,

E-mail: mariainesgiordano@hotmail.com

Palabras claves/Key word: formación docente. Enseñanza de las ciencias. Capacitación docente contínua/Teacher training. Enseñanza de las ciencias. Continuous teacher training.

Este trabajo relata una experiencia realizada por diferentes docentes como capacitación permanente. En el marco de "escribir textos de ciencias para el nivel terciario", se nos brindó la posibilidad de aprender a escribir ciencias, en el mas amplio sentido de la frase. Para llevar a cabo esta tarea y ver finalizada la obra, pasamos 1 año y varios meses trabajando en grupo, investigando, leyendo, escribiendo, releyéndonos y reescribiendo. El aporte de esta experiencia es inmensurable, sobre todo si de estrategias de enseñanza e investigación se trata. La producción de textos resulta sumamente rica por la cantidad de relaciones que por ella se traman, la génesis constante entre colegas, eruditos acompañantes, nivel de lectura, tipos de textos leídos (muchísimos para la enseñanza de la escritura, además de los científicos) y lo que todo esto deposita en el educador/autor. Los docentes que participamos del mismo, concluimos que la capacidad de brindar las clases específicas, su calidad, utilizando nuevos y mejores métodos de enseñanza aprendizaje es superior a las dictadas previamente; fuimos capaces de reconocer el transe en el proceso de E-A durante el periodo que llevamos a cabo la escritura; pudimos autoevaluarnos, ser críticos de nuestra propia labor, planificando un cómo mucho mejor. 
Bio - grafia. Escritos sobre la Biología y su Enseñanza. ISSN 2027

Edición Extraordinaria. p.p. 1147- 1154

Memorias del VIII Encuentro Nacional de Experiencias en Enseñanza de la Biología y la Educación Ambiental. III Congreso Nacional de Investigación en Enseñanza de la Biología.

En definitiva, el proceso de conversión de simples lectores a autores de texto es enriquecedor para mejorar la capacidad intelectual del docente en el tema investigado y sumar herramientas para el proceso diario dentro del aula.

El Instituto Nacional de Formación Docente (INFOD) desarrolló un dispositivo llamado Escritura en Ciencias, que contó con la participación de profesores de institutos de profesorados de todo el país. Éste tuvo una duración de 4 años aproximadamente. El mismo estuvo inspirado en un Programa del Sector Educación de la Oficina de UNESCO, Montevideo, denominado Docentes Aprendiendo en Red; auspiciado por éste y la Revista Ciencia Hoy. Gozó del apoyo de distinguidos especialistas referentes y colegas coordinadores de escritura y el Ministerio de Educación de la Nación. También se utilizó la adhesión, acompañamiento y promoción de las distintas asociaciones de profesores del país, ADBIA (Asociación de Docentes de Biología de Argentina), APFA (Asociación de Profesores de Fisca de Argentina) y ADEQRA (Asociación de Docentes de Enseñanza de la Química de la República Argentina).

\section{Cimientos del Proyecto}

- Trayecto de formación donde la experiencia y la práctica de escritura de los profesores participantes es colocada en primer plano.

- La propuesta se convierte en un espacio pensado para la realización de una tarea, escribir libros de ciencias, destinados a colegas docentes. No es un curso de escritura. No es una compilación de artículos.

- Un andamiaje conformado por los investigadores, los coordinadores y el aula virtual que acompaña desde el inicio hasta el final. Ejercicios vinculados al estudio y a la investigación sobre temas actuales de las ciencias naturales.

\section{Propósito del dispositivo}

Los profesores convocados se constituirán en autores de textos destinados a la formación docente.

Objetivos generales 
Bio - grafia. Escritos sobre la Biología y su Enseñanza. ISSN 2027

Edición Extraordinaria. p.p. 1147- 1154

Memorias del VIII Encuentro Nacional de Experiencias en Enseñanza de la Biología y la Educación Ambiental. III Congreso Nacional de Investigación en Enseñanza de la Biología.

- Promover la formación en escritura académica en los profesores de institutos de formación docente como una estrategia de generación y promoción del conocimiento científico.

- Transferir y difundir resultados de las producciones que se realicen, como resultado del proceso de grupos de estudio en el campo disciplinar de las ciencias naturales, como una manera de vincular procesos de escritura con prácticas investigativas y estrategias de enseñanza.

- Aportar un conjunto de producciones que aborden problemáticas vigentes en la agenda de las ciencias naturales, y que puedan servir de insumos y materiales de circulación acreditados entre los institutos de formación docente del país.

- Propiciar el trabajo colaborativo entre los participantes y desarrollar estrategias conjuntas de escritura profesional.

\section{En cuanto a la escritura}

Escritura como práctica situada: Leer y escribir son prácticas situadas que se aprenden a través de su puesta en funcionamiento generando reflexiones sobre su uso. Conjunto de estrategias que incluyen la formación de adultos, el acompañamiento y la escritura profesional. Interiorización de saberes y herramientas culturales; Modos de posicionamiento enunciativo propios de las comunidades de referencia. (Bernié, 2004).

Comunidades discursivas y dispositivos de formación: Modelos teóricos vigentes que permiten hoy pensar la escritura y los procesos de escritura profesional en su complejidad. Aportes de las teorías cognitivista y sociocultural: comunidades de prácticas y comunidades discursivas.

Mediación de procesos de conocimientos: La escritura como una mediación relevante para los procesos de conocimiento. La escritura concebida como un trabajo intelectual (ensayos, intentos, revisiones, rectificaciones), la escritura como un aprendizaje en cada nuevo contexto que la demanda. (Bereiter, 1992; Chartrand, S. 2005)

Acompañamiento: El desarrollo de prácticas de escritura requiere condiciones para sostener un proceso completo: prácticas propias de una comunidad discursiva específica: diferentes formas del acompañamiento, la puesta en escena de situaciones comunicativas: diálogo con otros, con los pares, reflexiones sobre la experiencia y orientaciones expertas. (Paul, M. 2002, Bernié, 2004) 
Bio - grafia. Escritos sobre la Biología y su Enseñanza. ISSN 2027

Edición Extraordinaria. p.p. 1147- 1154

Memorias del VIII Encuentro Nacional de Experiencias en Enseñanza de la Biología y la Educación Ambiental. III Congreso Nacional de Investigación en Enseñanza de la Biología.

La necesidad del mismo surge en suplir la ausencia de los libros de textos específicos para la formación docente, con ejemplos actuales y de la región/país. La convocatoria para el mismo se realiza a nivel nacional, a través de los institutos de formación docente para aquellos docentes interesados en participar de estas experiencias. Se editaron 6 libros por cohorte. Los respectivos títulos y referentes científicos fueron:

- $1^{\circ}$ cohorte 2010-2011:

\begin{tabular}{|c|l|}
\hline $\begin{array}{c}\text { Los plaguicidas, aquí y ahora } \\
\text { H20 en estado vulnerable }\end{array}$ & Dr. Raúl Alzogaray \\
\hline Del gen a la proteína & $\begin{array}{l}\text { Dr. Alberto Kornblihtt - Dr. } \\
\text { Manuel Muñoz }\end{array}$ \\
\hline La multiplicidad de la vida & Dr. Jorge Crisci \\
\hline $\begin{array}{c}\text { Cerebro y memoria } \\
\text { La evolución biológica, actualidad y } \\
\text { debates }\end{array}$ & Dra. Noel Federman \\
\hline
\end{tabular}

- $2^{\circ}$ cohorte 2011-2012:

\begin{tabular}{|c|c|}
\hline Ecosistemas terrestres & Dr. Rolando León \\
\hline Ecosistemas acuáticos & Dr. Juan Gappa \\
\hline El big bang y la física del cosmos & Dr. Alejandro Gangui \\
\hline Cambio climático & Dra. Marcela González \\
\hline
\end{tabular}


Bio - grafia. Escritos sobre la Biología y su Enseñanza. ISSN 2027

Edición Extraordinaria. p.p. 1147- 1154

Memorias del VIII Encuentro Nacional de Experiencias en Enseñanza de la Biología y la Educación Ambiental. III Congreso Nacional de Investigación en Enseñanza de la Biología.

\begin{tabular}{|c|c|}
\hline Energía:característicasy contextos & Dr. José Natera \\
\hline Epidemias y salud pública & Dr. Mario Lozano \\
\hline
\end{tabular}

- $3^{\circ}$ cohorte 2013-2014:

\begin{tabular}{|c|c|}
\hline $\begin{array}{r}\text { Biotecnología: el poder del laboratorio } \\
\text { Convergencia: Electrónica, informática y } \\
\text { telecomunicaciones }\end{array}$ & Ing. Carlos Palotti \\
\hline $\begin{array}{r}\text { Nanotecnología hoy: el desafío de conocer y y } \\
\text { enseñar }\end{array}$ & Dr. Galo Soler Illia \\
\hline Alimentos: historia, presente y futuro & Dra. Laura Malec \\
\hline Las radiaciones y la energía del universo & Dr. Jorge Torga \\
\hline Los movimientos del planeta Tierra & Dr. Silvio Peralta \\
\hline
\end{tabular}

Cada libro contó con la presencia de de 5 autores, los cuales se encargaron de la escritura de un capítulo. A partir de la $2^{\circ}$ cohorte se anexó a los libros un capítulo final de aplicaciones didácticas específicas de cada tema; en la $3^{\circ}$ y última cohorte, además, se sumó el seguimiento longitudinal de casos, un autor por libro, seleccionado al azar y una guía personalizada de autoevaluación. La realización de los mismos se llevó a cabo a través del Aula Virtual (plataforma virtual) y de 5 encuentros durante el transcurso del año de escritura. Los talleres presenciales se llevaron a cabo en CABA (Ciudad Autónoma de Buenos Aires), fueron intensivos, con una duración de 2 días, a mitad de semana (cuyas inasistencias en las escuelas estuvieron justificadas por el Ministerio), de 10 horas de 
Bio - grafia. Escritos sobre la Biología y su Enseñanza. ISSN 2027

Edición Extraordinaria. p.p. 1147- 1154

Memorias del VIII Encuentro Nacional de Experiencias en Enseñanza de la Biología y la Educación Ambiental. III Congreso Nacional de Investigación en Enseñanza de la Biología.

trabajo cada jornada. En el primer encuentro se presentaron las distintas charlas que los investigadores seleccionados prepararon para el mismo y la presentación de los escritores de cada libro con una simple actividad práctica; se delimitó el tema-inicio, previsto desde el momento de la inscripción al concurso. En el segundo encuentro se armó un bosquejo del índice y se distribuyeron los capítulos entre los docentes escritores; el tercer encuentro encontró a los docentes con un arduo trabajo previo en la plataforma virtual, para poder en éste momento, realizar los primeros borradores, revisiones conceptuales e intercambio entre pares. Durante el cuarto encuentro se comenzó con el capítulo didáctico, hubieron más devoluciones de los textos por parte, tanto de los investigadores como los colegas docentes y la referente de escritura. El último encuentro estuvo plasmado de emociones, nerviosismo y tensión, ya que aquí se revieron los capítulos pensando en su ulterior edición, ajustando además, la agenda final.

En cuanto a la disciplina de cada docente escritor, podemos decir que los interesados-convocados (esto es directamente proporcional a los temas pensados previamente a editar, de acuerdo a las estadísticas de profesorados en el país-temas a escribir) fueron aquellos de Biología, mientras que los profesores de Física siguieron en cantidad y con un porcentaje levemente menor, los profesores de Química.

Las dimensiones de escritura que atravesó este proyecto estuvieron dadas por un constante feed-back entre docentes escritores-moderadores de enseñanza-científicodocentes escritores, produciendo un trabajo constante de escritura-lectura-relecturaautolectura y sus correspondientes correcciones. Por ejemplo:

- Devolución y orientación del coordinador al/a autor/a.

- Reformulación posterior, luego de la devolución.

- Proceso reflexivo producido por las devoluciones y reformulaciones.

- Resultado de la devolución: proceso reflexivo de autoescritura.

- Resultado de la actividad:

- Relación título-contenido-lector posible.

- Objetivos del capítulo; coherencia entre ellos con su título, con su contenido y con el resto del libro.

- Comprensión, ambigüedad, complejidad del texto, entre otros. 
Bio - grafia. Escritos sobre la Biología y su Enseñanza. ISSN 2027

Edición Extraordinaria. p.p. 1147- 1154

Memorias del VIII Encuentro Nacional de Experiencias en Enseñanza de la Biología y la Educación Ambiental. III Congreso Nacional de Investigación en Enseñanza de la Biología.

- Restricciones en el texto por requerimientos editoriales generales.

Los diferentes momentos de escritura fueron divididos en:

- Diseño y planificación: compuesta por la escritura y procesos de conocimiento. Escritura-revisión-reescritura.

- Diseñar plan de obra según posible lector y temática propuesta y la adecuación al plan diseñado: búsqueda y lectura de fuentes bibliográficas. Devolución de correcciones constructivas hacia los pares.

Fueron utilizados diferentes recursos y materiales, como se dijo anteriormente, la plataforma virtual, donde visualizar las conferencias brindadas por los investigadores, otros trabajos de interés seleccionados por ellos, de propia autoría o ajena, libros en formatos digital (http://portales.educacion.gov.ar/infd/escritura-en-ciencias/); esta plataforma también se utilizó como contacto on line entre los investigadores, los referentes de escritura y los propios colegas, a través de mails, foros y grupos de trabajo. En ella encontrábamos la documentación obligatoria a leer, algunos documentos relevantes para el taller de lectura y la futura investigación preescritura.

Todos los libros contienen una Presentación general del dispositivo realizada por el equipo de trabajo del INFOD, una Introducción donde se comenta brevemente el tema tratado en el libro, realizado por el grupo de docentes escritores, los Capítulos meramente del tema y Palabras Finales (en $1^{\circ}$ cohorte) o con una reflexión epistemológica sobre la enseñanza en el nivel secundario y superior, llamados por tal motivo: "capítulos didácticos". En ellos se representan diversos enfoques de estrategias de enseñanza de las ciencias, entre los que podemos encontrar, analogías y modelos (enseñanza y aprendizaje a través de representaciones y modelos), experimentos (enseñanza de la ciencia y diseños experimentales), el laboratorio escolar, integración con Tic, experiencias de aprendizajes in situ (trabajo en campo), experiencias de aprendizajes fuera de la escuela (museos, parques, observatorios). 
Bio - grafia. Escritos sobre la Biología y su Enseñanza. ISSN 2027

Edición Extraordinaria. p.p. 1147- 1154

Memorias del VIII Encuentro Nacional de Experiencias en Enseñanza de la Biología y la Educación Ambiental. III Congreso Nacional de Investigación en Enseñanza de la Biología.

Bibliografía consultada:

Caríssimo, M. S. et.al. (2013). Ecosistemas acuáticos - 1a ed. - Buenos Aires : Ministerio de Educación de la Nación. Escritura en ciencias; 8. ISBN 978-950-00-0987-4

Bibliografía on line:

http://cedoc.infd.edu.ar/upload/08Ecosistemas_acuaticos.pdf 